\title{
3-S36-4 Symposium36
}

\section{Systems Biology of Mammalian Sleep/Wake Cycles Phosphorylation Hypothesis of Sleep}

\author{
Hiroki R. Ueda ${ }^{1,2}$ \\ ${ }^{\text {I} G r a d u a t e ~ S c h o o l ~ o f ~ M e d i c i n e ~ U T o k y o, ~}{ }^{2}$ BDR RIKEN
}

The detailed molecular and cellular mechanisms underlying NREM sleep (slow-wave sleep) and REM sleep (paradoxical sleep) in mammals are still elusive. To address these challenges, we first constructed a mathematical model, Averaged Neuron Model (AN Model), which recapitulates the electrophysiological characteristics of the slowwave sleep. Comprehensive bifurcation analysis predicted that a $\mathrm{Ca}^{2+}$-dependent hyperpolarization pathway may play a role in slow-wave sleep. To experimentally validate this prediction, we generate and analyze $26 \mathrm{KO}$ mice, and found that impaired $\mathrm{Ca}^{2+}$-dependent $\mathrm{K}^{+}$channels (Kcnn2 and Kcnn3), voltage-gated $\mathrm{Ca}^{2+}$ channels (Cacna1g and Cacna1h), or $\mathrm{Ca}^{2+} /$ calmodulin-dependent kinases (Camk2a and Camk2b) decrease sleep duration, while impaired plasma membrane $\mathrm{Ca}^{2+}$ ATPase (Atp2b3) increases sleep duration. Genetical (Nr3a) and pharmacological intervention (PCP, MK-801 for Nr1/Nr2b) and whole-brain imaging validated that impaired NMDA receptors reduce sleep duration and directly increase the excitability of cells. Based on these results, we propose phoshporylation hypothesis of sleep that phosphorylation-dependent regulation of $\mathrm{Ca}^{2+}$-dependent hyperpolarization pathway underlies the regulation of sleep duration in mammals. We also recently developed a simplified mathematical model, Simplified Averaged Neuron Model (SAN Model), which uncover the important role of $\mathrm{K}^{+}$leak channels in NREM sleep. In this talk, I will also describe how we identify essential genes (Chrm1 and Chrm3) in REM sleep regulation, and propose a plausible molecular definition of a paradoxical state of REM sleep. 